\title{
Reconstruyendo la diferencia de camino óptico de un interferograma real degradado por ruido mediante la solución de diferentes problemas de optimización
}

\author{
J. J. Sánchez-Escobar ${ }^{a}$, L. I. Barbosa-Santillán ${ }^{b}$, J. Castro-Ramos ${ }^{c}$ y L. F. Barbosa-Santillán ${ }^{d}$ \\ ${ }^{a}$ Centro de Enseñanza Técnica Industrial, \\ Nueva Escocia 1885, Fraccionamiento Providencia 5a Sección, 44638, Guadalajara, Jalisco, México. \\ ${ }^{b}$ Universidad de Guadalajara, CUCEA-Departamento de Sistemas de Información, \\ Periférico Norte $N^{\circ}$ 799, Núcleo Universitario Los Belenes, 45100, Zapopan, Jalisco, México. \\ ${ }^{c}$ Instituto Nacional de Astrofísica, Óptica y Electrónica, \\ Luis Enrique Erro $N^{\circ}$ 1, 72840, Tonantzintla, Puebla, México. \\ ${ }^{d}$ Universidad Tecnológica de Puebla, \\ Antiguo Camino a la Resurrección N 1002-A, Zona Industrial Puebla Oriente, 72300, Puebla, Puebla, México.
}

Received 3 July 2020; accepted 13 February 2021

\begin{abstract}
Este artículo presenta un estudio numérico comparativo que demuestra la factibilidad de la optimización estocástica (OE) para reconstruir la diferencia de camino óptico (DCO) a partir de un interferograma real degradado por ruido, ya sea por la maximización del coeficiente de correlación o por la minimización de la distancia euclidiana donde la optimización conseguida para cada función objetivo corresponde a la solución más cercana al óptimo global, sin ser dominada por un óptimo local. Con la finalidad de mostrar la eficacia de diferentes algoritmos de OE, inspirados en cómputo evolutivo, nosotros proponemos una solución al problema de maximización mediante el uso de un algoritmo genético con las aberraciones primarias descritas por Kingslake, mientras que para la solución del problema de minimización se propone una estrategia evolutiva con los polinomios de Zernike. Los resultados numéricos muestran la sencillez, robustez y precisión de ambos algoritmos de optimización para calcular sus correspondientes coeficientes de aberración. De esta forma, este trabajo ofrece una oportunidad idónea para integrar las habilidades adquiridas por los estudiantes universitarios de ciencias e ingeniería en materias como metrología óptica interferométrica, métodos numéricos y programación con el propósito de realizar análisis de interferogramas.
\end{abstract}

Descriptores: Reconstrucción numérica; problemas inversos; optimización estocástica; experimentos de demostración para estudiantes no graduados; estudiantes no graduados de ciencias e ingeniería.

This paper presents a comparative numerical study that demonstrates the feasibility of stochastic optimization (SO) for reconstructing the optical path difference (OPD) from a real interferogram degraded by noise, either by the maximization of the correlation coefficient or by the minimization of the Euclidean distance where the optimization achieved for each objective function corresponds to the near-optimal solution without being dominated by a local optimum. In order to show the efficacy of different SO algorithms based on evolutionary computation, we propose a solution to the maximization problem by using a genetic algorithm with the primary aberrations described by Kingslake, while for the solution of the minimization problem an evolutionary strategy with Zernike polynomials is proposed. The numerical results show the simplicity, robustness, and accuracy of both SO algorithms to calculate their corresponding aberration coefficients. Thus, this work offers an ideal opportunity to integrate the skills acquired by university students of science and engineering in subjects such as interferometric optical metrology, numerical methods, and programming with the purpose of performing interferogram analysis.

Keywords: Numerical reconstruction; inverse problems; stochastic optimization; demonstration experiments for undergraduates; scienceengineering undergraduate students.

PACS: 42.30.Wb; 02.30.Zz; 02.60.Pn; 01.50.My; 01.30.1b.

DOI: https://doi.org/10.31349/RevMexFisE.18.020207

\section{Introducción}

Por ser herramientas de precisión, los instrumentos ópticos, tales como microscopios, telescopios, cámaras fotográficas, etc., son fabricados con una alta calidad óptica. Las desviaciones del contorno correcto en las superficies de una lente individual, de fracciones de una micra, pueden resultar en un funcionamiento incorrecto del instrumento final [1]. Las pruebas de diagnóstico más comúnmente usadas para medir la desviación en una superficie óptica son aquellas que se basan en interferometría óptica [1-2].
Un interferómetro óptico, de división de amplitud por transmisión-reflexión del tipo Fizeau o Twyman-Green, genera un interferograma a través de la superposición de dos frentes de onda donde uno de ellos corresponde al frente de onda conocido, el cual es llamado frente de onda de referencia y es, en general, un frente de onda ideal plano o esférico, mientras que el otro, el de prueba, representa al frente de onda degradado o aberrado por la diferencia de camino óptico $(D C O)$. De este modo, la $D C O$, entre los dos frentes de onda, determina la desviación de la superficie óptica de prueba de la superficie óptica ideal de referencia [1-2]. 
Por lo tanto, al usar un interferómetro óptico, del tipo Fizeau o Twyman-Green, con la finalidad de determinar la calidad óptica de una superficie óptica, se hace necesario no solamente capturar la imagen digital del interferograma en tonos de gris a través del uso de una cámara CCD, sino también almacenar en la computadora dicha imagen digital, de mxn píxeles, para el análisis de los niveles de gris en cada posición $(x, y)$ del arreglo de píxeles y así reconstruir la información cuantitativa de la $D C O$, la cual concierne a los defectos de la superficie óptica bajo prueba [3-6].

Para el análisis de un interferograma digitalizado, es necesario considerar la expresión del interferograma detectado por una cámara CCD en un plano $x y$. Dicha expresión es definida por [1-7]:

$$
\begin{aligned}
I_{d}(x, y) & =\left(a(x, y)+b(x, y) \cos \left[\frac{2 \pi}{\lambda} D C O(x, y)\right]\right) \\
& +n(x, y),
\end{aligned}
$$

en donde $a(x, y)$ y $b(x, y)$ son funciones que representan la iluminación de fondo, así como el contraste local, respectivamente, $\lambda$ es la longitud de onda de la luz, $D C O(x, y)$ corresponde a la diferencia de camino óptico y $n(x, y)$ representa el ruido introducido de un modo aditivo y/o multiplicativo [7-9]. Además, las funciones de iluminación de fondo y de contraste local contienen las intensidades de los campos que interfieren, superpuestos, así como diferentes tipos de fluctuaciones aleatorias que degradan a $I_{d}$. La función $a(x, y)$ contiene las contribuciones aditivas, tales como las variaciones de iluminación, los cambios de reflectividad, el ruido electrónico y la difracción de partículas de polvo en los caminos ópticos, mientras que la función $b(x, y)$ se asocia a las influencias multiplicativas, las cuales incluyen la razón entre los haces de luz de referencia y prueba, la decorrelación de moteado, así como las variaciones de contraste ocasionadas por la formación de moteado [7-9].

También cabe mencionar que la función $D C O$, en la Ec. (1), puede ser expresada en términos de las aberraciones primarias descritas por Kingslake, las cuales pueden ser representadas de la siguiente manera [2]:

$$
\begin{aligned}
D C O(x, y) & =A x+B y+C\left(x^{2}+y^{2}\right)+D\left(x^{2}+3 y^{2}\right) \\
& +E y\left(x^{2}+y^{2}\right)+F\left(x^{2}+y^{2}\right)^{2},
\end{aligned}
$$

en donde $A$ es una inclinación sobre el eje $x, B$ es una inclinación sobre el eje $y, C$ es el coeficiente de foco, $D$ es el coeficiente de astigmatismo, $E$ es el coeficiente de coma y $F$ es el coeficiente de aberración esférica. Adicionalmente, todos los coeficientes son expresados como múltiplos de $\lambda$.

De igual forma que en la ecuación anterior, la función $D C O$ puede ser definida por los polinomios de Zernike (PZ), los cuales son detallados en la descripción proporcionada en la Tabla I [10].

Es importante destacar que en publicaciones previas han sido reportadas diferentes técnicas de optimización estocástica, conocidas como algoritmos evolutivos (AEs), para su uso en la reconstrucción de la función $D C O$ a partir de un interferograma digitalizado $I_{d}$ [11-13]. En todos los casos reportados, la función objetivo es definida como una expresión que indica la medida de similitud que existe entre dos imágenes en tonos de gris. Dicha función objetivo usada por cualquiera de los AEs propuestos, ya sea una estrategia evolutiva o un algoritmo genético, es expresada de forma general como [11-13]:

$$
f\left(I, I_{d}\right)=\varphi,
$$

en donde $I$ corresponde al interferograma simulado con la Ec. (1), descartando $n(x, y)$, y cuyos coeficientes de aberración, a través de la Ec. (2) o la Tabla I, definen la medida de similitud $\varphi$ que existe entre $I$ y el interferograma digitalizado $I_{d}$, siendo $\varphi$ un número real positivo.

La optimización de la Ec. (3), independientemente del AE usado, es inicializada con un conjunto de soluciones o individuos generados aleatoriamente donde cada individuo es definido como el vector de un conjunto indexado de elementos o coeficientes de aberración acorde a la Ec. (2) o la Tabla I. El tamaño de la población de individuos es mantenido constante en las sucesivas iteraciones del proceso de optimización. Además, cada interferograma $I$, simulado a partir de su correspondiente individuo, es evaluado por la Ec. (3) para establecer su valor de aptitud en términos de $\varphi$. Por lo tanto, durante cada iteración del proceso, aquellos individuos con buenos valores de aptitud tienen una mayor probabilidad de que en la siguiente iteración evolucionen hacia mejores soluciones a través del uso de procesos estocásticos de recombinación, mutación y selección.

La optimización de la Ec. (3) es finalizada cuando en una determinada iteración es encontrado un individuo $a_{\mathrm{opt}}$, cuyos coeficientes de aberración definen un valor de aptitud $\varphi_{\text {opt }}$ que corresponde a la solución más cercana al optimo global, es decir, $I_{\mathrm{opt}}$ es obtenido con un valor óptimo de similitud $\varphi_{\text {opt }}$ respecto a $I_{d}$. De esta manera la función $D C O_{\text {opt }}$ que define a $I_{\mathrm{opt}}$, en términos de la Ec. (2) o los PZ en la Tabla I, es reconstruida con precisión y exactitud, a pesar de que $I_{d}$ es degradado por ruido aditivo y/o multiplicativo. Cada uno de los AEs propuestos para la optimización de la Ec. (3), se diferenció por la manera de implementar los procesos estocásticos antes mencionados [11-13].

En el trabajo reportado en la Ref. [11], una estrategia evolutiva es usada en el análisis de diferentes interferogramas simulados acorde a la Ec (1), los cuales son degradados por ruido Gaussiano cuya densidad de probabilidad obedece a una distribución normal. Este tipo de fluctuación aleatoria, relacionado al ruido electrónico [7-9], es generado sobre cada franja del interferograma analizado siguiendo dos propiedades: Una media igual a cero y una desviación estándar igual a $\sigma_{m}$ [11]. Partiendo de esta condición, la Ec. (3) es optimizada usando la estrategia evolutiva con la formulación de Kingslake establecida en la Ec. (2), obteniendo al final del proceso de optimización la mínima distancia euclidiana entre $I_{\mathrm{opt}} \mathrm{y}$ $I_{d}$ cuyo valor $\varphi_{\mathrm{opt}}$ correspondió a la solución más cercana 
al mínimo global. Los resultados numéricos mostraron la robustez y eficacia de la estrategia evolutiva para reconstruir la función $D C O$ en términos de las aberraciones primarias descritas en la Ec. (2), incluso cuando la degradación es muy notoria en los interferogramas analizados.

Resultados similares a los reportados en la Ref. [11], en robustez y eficacia, han sido obtenidos con un algoritmo genético [12], así como por una estrategia evolutiva durante el análisis de interferogramas reales afectados por ruido [13]. En todos los experimentos numéricos realizados con los dos AEs, la función objetivo minimizada y la función $D C O$ reconstruida son definidas por la distancia euclidiana y los PZ descritos en la Tabla I, respectivamente.

Teniendo en consideración que recientemente en un estudio numérico el coeficiente de correlación ha sido propuesto para comparar la similitud entre dos interferogramas acorde a la descripción proporcionada en la Ec. (3) [14], en este trabajo se presenta un experimento numérico comparativo que demuestra la eficacia de la optimización estocástica para reconstruir la función $D C O$ de un interferograma real degradado por ruido $\left(I_{d}\right)$, ya sea maximizando el coeficiente de correlación o minimizando la distancia euclidiana, evitando, en cualquiera de los dos procesos de optimización propuestos, la convergencia prematura a un óptimo local. Por lo tanto, para resolver el problema de maximización nosotros proponemos el uso de un algoritmo genético con la formulación de Kingslake descrita en la Ec. (2), mientras que para resolver el problema de la minimización se propone el uso de una estrategia evolutiva con los PZ mencionados en la Tabla I.

Los resultados numéricos dan evidencia que cada AE propuesto posee la capacidad de encontrar el número óptimo de términos polinomiales requeridos en la reconstrucción de su respectiva función $D C O$, así como de obtener los valores óptimos de sus correspondientes coeficientes de aberración. Por consiguiente, cada interferograma simulado resultante $I_{\mathrm{opt}}$, optimizado en términos de su respectiva función $D C O_{\text {opt }}$, es prácticamente idéntico al interferograma analizado $I_{d}$.

El artículo es estructurado de la siguiente forma: En la Sec. 2 es proporcionada una descripción generalizada de los
AEs considerados para el estudio numérico comparativo. En la Sec. 3, son mostradas las discusiones en torno al análisis de cada $I_{d}$ propuesto. Finalmente, las principales conclusiones son presentadas en la Sec. 4.

\section{Algoritmos propuestos para reconstruir la función $D C O$ desde un $I_{d}$ mediante la opti- mización de diferentes funciones objetivo}

Es importante resaltar que para encontrar la solución de un problema inverso, como es la reconstrucción de la función $D C O$ a partir de $I_{d}$ en la Ec. (1), conviene que dicho problema sea replanteado como un problema de optimización, pero en ocasiones una función objetivo puede tener una forma muy compleja o su expresión analítica puede ser desconocida, de tal modo que el problema de optimización resulte difícil o imposible de resolver a través del uso de técnicas tradicionales de búsqueda y optimización [15]. Por otro lado, un AE, en cualquiera de las dos versiones mencionadas en el capítulo anterior, no requiere información de la derivada de la función objetivo, razón por la cual solo necesita del uso de una función objetivo, como la descrita en la Ec. (3), con la finalidad de calcular durante cada iteración los valores de aptitud de su población de soluciones [11-13]. La razón por la que un AE encuentra la solución más cercana un óptimo global sin ser afectado por el ruido, se debe a que en cada iteración el algoritmo ejecuta en el espacio de muestreo una búsqueda en paralelo de aquellas soluciones con buenos valores de aptitud [11-13]. Dicha búsqueda simultanea es realizada a través de procesos de transición probabilísticos, los cuales son conocidos como procesos de recombinación, mutación y selección [16-18].

Con la finalidad de implementar el experimento numérico comparativo con los AEs mencionados en la primera sección, las siguientes funciones objetivo, el coeficiente de correlación $(C O R R)$ y la distancia euclidiana $(D E)$, deben ser optimizadas:

$$
\begin{aligned}
\operatorname{CORR}\left(I_{k}, I_{e c}\right) & =\frac{\sum_{i=1}^{m} \sum_{j=1}^{n}\left(I_{k_{i, j}}-\bar{I}_{k}\right)\left(I_{e c_{i, j}}-\bar{I}_{e c}\right)}{\sqrt{\left(\sum_{i=1}^{m} \sum_{j=1}^{n}\left[I_{k_{i, j}}-\bar{I}_{k}\right]^{2}\right)\left(\sum_{i=1}^{m} \sum_{j=1}^{n}\left[I_{e c}-\bar{I}_{e c}\right]^{2}\right)}}=\varphi_{1}, \\
D E\left(I_{k}, I_{e c}\right) & =\sqrt{\sum_{i=1}^{m} \sum_{j=1}^{n}\left(I_{k_{i, j}}-I_{e c_{i, j}}\right)^{2}}=\varphi_{2} .
\end{aligned}
$$

En las Ecs. (4) y (5), $I_{e c}$ corresponde al interferograma real $I_{d}$ cuyo histograma de niveles de intensidad de gris es ecualizado, con el fin de aumentar el rango de niveles de gris de 0 a 255, debido a que dicho interferograma es degradado por ruido. Por lo tanto, en ambas funciones objetivo, $I_{k}$ puede ser comparado con $I_{e c}$ para establecer los valores de aptitud en $\varphi_{1} \mathrm{y} \varphi_{2}$, ya que $I_{k}$ es simulado en el rango de 256 tonos de gris acorde a la Ec. (1), sin considerar ruido añadido $n(x, y)$. 


$$
g=0
$$

inicializa $\mathcal{P}(0)$;

mientras (las condiciones de terminación no sean cumplidas) hacer

$$
\begin{aligned}
& \text { recombinación y mutación a } \mathcal{P}(0) \text { para producir } \mathcal{C}(0) \\
& g=g+1 \\
& \mathcal{P}(g) \text { es obtenida seleccionando a los } \mu \text { individuos con altos valores } \\
& \text { de aptitud de } \mathcal{P}(0) \cup \mathcal{C}(0) ;
\end{aligned}
$$

fin

FIGURA 1. Procedimiento para optimizar una función objetivo con un AE.

Además, $I_{k}$ en la Ec. (4) es simulado a partir de su solución candidato o individuo $a_{k}$, el cual corresponde al vector de un conjunto indexado de elementos que toman valores en número reales, siendo estos elementos relacionados a los coeficientes de aberración de la formulación de Kingslake en la Ec. (2), y en donde $k \in\{1, \ldots, \mu\}$. Así también en la Ec. (4), $\bar{I}_{e c}$ y $\bar{I}_{k}$ representan el promedio de los niveles de intensidad de los respectivos interferogramas. Por otro lado, en la Ec. (5), el interferograma simulado $I_{k}$ es obtenido a partir de su respectivo individuo $a_{k}$ cuyo vector con su conjunto indexado de elementos, en números reales, es definido por los coeficientes de aberración de los PZ expresados en la Tabla I.

En la Fig. 1 se muestra un pseudocódigo con los procesos estocásticos de recombinación, mutación y selección que dan forma a un $\mathrm{AE}$, ya sea en la versión de estrategia evolutiva o de algoritmo genético. La población, en la iteración o generación $\mathscr{G}$, está representada por la variable $\mathcal{P}$ dependiente de $\mathscr{g}$, siendo $\mathcal{P}(0)$ la población inicial de soluciones o individuos generados aleatoriamente. Un AE inicia la optimización de la función objetivo, como la descrita en la Ec. (3), usando la población $\mathcal{P}(0)$ e implementa una búsqueda simultánea de soluciones potenciales en regiones del espacio de muestreo aplicando los procesos estocásticos mencionados anteriormente con el propósito de formar una nueva población $\mathcal{P}(\mathscr{g})$ en $\mathscr{g}=\mathscr{g}+1$. La aplicación de tales procesos estocásticos en las siguientes generaciones da como resultado que el AE encuentre nuevos conjuntos de individuos que correspondan a mejores soluciones dentro del espacio de muestro. El proceso de optimización finaliza cuando un número predeterminado de generaciones es obtenido o cuando la función objetivo que es optimizada por el individuo $a_{k_{\text {opt }}}$, en $\mathcal{P}(\mathcal{F})$, es igual al valor objetivo predeterminado $\varphi_{k_{\mathrm{opt}}}$, en donde $a_{k_{\mathrm{opt}}}$ corresponde a la solución más cercana al óptimo global.

El primer AE, propuesto para maximizar a la Ec. (4), está basado en un algoritmo genético (AG) previamente usado en la solución de un problema de minimización [12], no obstante, en el presente trabajo nuestro AG es convenientemente modificado en la función objetivo y en la función $D C O$, con el propósito de implementar otra aplicación exitosa de dicho algoritmo de optimización estocástica.

Una descripción detalla del AG puede ser encontrada en [12]. En la maximización de la Ec. (4), los datos de entrada al AG son definidos por $I_{e c}$ y dicho algoritmo arroja como datos de salida al individuo $a_{k_{\mathrm{opt}}}$ que representa a los valores de los coeficientes de aberración descritos en la Ec. (2), los cuales definen a la función $D C O_{\text {opt }}$ que mejor se asocia a la información codificada en $I_{e c}$. A continuación, es proporcionada una descripción del AG, especificando las características particulares que poseen sus procesos estocásticos en la maximización de la Ec. (4):

Paso 1: Una población inicial de individuos, $\mathscr{F}=0$, es generada aleatoriamente:

$$
\mathcal{P}_{\text {padres }}(0)=\left\{a_{1}(0), \ldots \ldots, a_{\mu}(0)\right\},
$$

en donde cada individuo $a_{k}$ representa a los 6 coeficientes de aberración de la Ec. (2):

$$
a_{k}=\left[x_{k, 1}, \ldots \ldots, x_{k, 6}\right], \quad k \in\{1, \ldots \ldots, \mu\} .
$$

Paso 2: La aptitud $\varphi_{1_{k}}$ de cada individuo $a_{k}$ es evaluada con la función objetivo $\operatorname{COR} R\left(I_{k}, I_{e c}\right)$ expresada en la Ec. (4).

Paso 3: Las aptitudes de todos los individuos son agrupadas en orden descendente en un vector fila, del mayor valor $\varphi_{1_{1}}$ al menor valor $\varphi_{1_{\mu}}$ :

$$
\text { aptitudes }=\left[\varphi_{1_{1}}, \ldots \ldots, \varphi_{1_{\mu}}\right]
$$

en donde $\varphi_{1_{1}} \mathrm{y} \varphi_{1_{\mu}}$ representan al mejor y al peor individuo, respectivamente. El conjunto de padres que son considerados para el siguiente paso, son aquellos cuyos valores de aptitud son definidos en el intervalo que va de $\varphi_{1_{1}}$ a $\varphi_{1_{\mu / 2}}$. El resto de la población es excluida del proceso de optimización.

Paso 4: Una aproximación de rueda de la ruleta es aplicada a la población resultante: $P_{\text {padres }}(0)=b_{1}(0), \ldots \ldots, b_{\mu / 2}(0)$. La finalidad de esta aproximación es la de seleccionar aleatoriamente un conjunto de pares de individuos a partir de dicha población, en donde la cantidad de pares de individuos seleccionados viene definida por $\mu / 4$. El proceso comienza

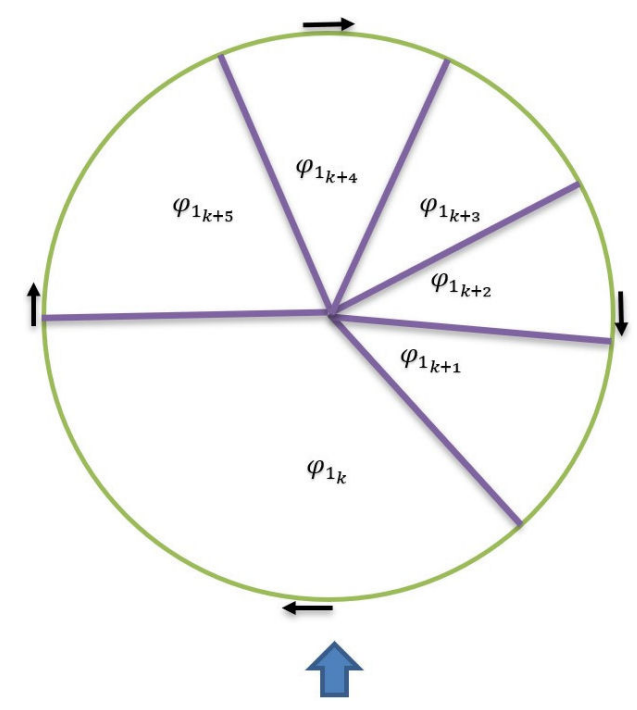

FIGURA 2. Ejemplo ilustrativo de la rueda de ruleta considerando 6 individuos. Al finalizar el giro de la rueda, la probabilidad de que el individuo $b_{k}(0)$ sea elegido es mayor que la de los otros individuos. 
TABLA I. Polinomios de Zernike con sus respectivos coeficientes de aberración. Cada coeficiente es expresado como múltiplo de la longitud de onda usada en el análisis de un interferograma. Los coeficientes de $q_{0}$ a $q_{3}$ no representan las aberraciones producidas por una superficie óptica. El coeficiente $q_{0}$ define el valor medio de la $D C O$ en la pupila del interferómetro, mientras que $q_{1}, q_{2}$ y $q_{3}$ corresponden a los errores de posicionamiento, de inclinación y traslación, que pueden tener las componentes del arreglo óptico interferométrico, tales como las superficies ópticas de prueba y de referencia.

\begin{tabular}{|c|c|c|c|}
\hline Término & Coeficiente & Polinomio de Zernike & Descripción \\
\hline 0 & $q_{0}$ & 1 & Piston \\
\hline 1 & $q_{1}$ & $\rho \cos \theta$ & Tilt $\mathrm{x}$ \\
\hline 2 & $q_{2}$ & $\rho \sin \theta$ & Tilt y \\
\hline 3 & $q_{3}$ & $2 \rho^{2}-1$ & Defoco \\
\hline 4 & $q_{4}$ & $\rho^{2} \cos 2 \theta$ & Astigmatismo $\mathrm{x}$ \\
\hline 5 & $q_{5}$ & $\rho^{2} \sin 2 \theta$ & Astigmatismo y \\
\hline 6 & $q_{6}$ & $\left(3 \rho^{2}-2\right) \rho \cos \theta$ & Coma $\mathrm{x}$ \\
\hline 7 & $q_{7}$ & $\left(3 \rho^{2}-2\right) \rho \sin \theta$ & Coma y \\
\hline 8 & $q_{8}$ & $6 \rho^{4}-6 \rho^{2}+1$ & Esférica primaria \\
\hline 9 & $q_{9}$ & $\rho^{3} \cos 3 \theta$ & Trébol x \\
\hline 10 & $q_{10}$ & $\rho^{3} \sin 3 \theta$ & Trébol y \\
\hline 11 & $q_{11}$ & $\left(4 \rho^{2}-3\right) \rho^{2} \cos 2 \theta$ & Astigmatismo secundario $\mathrm{x}$ \\
\hline 12 & $q_{12}$ & $\left(4 \rho^{2}-3\right) \rho^{2} \sin 2 \theta$ & Astigmatismo secundario y \\
\hline 13 & $q_{13}$ & $\left(10 \rho^{4}-12 \rho^{2}+3\right) \rho \cos \theta$ & Coma secundaria $\mathrm{x}$ \\
\hline 14 & $q_{14}$ & $\left(10 \rho^{4}-12 \rho^{2}+3\right) \rho \sin \theta$ & Coma secundaria y \\
\hline 15 & $q_{15}$ & $20 \rho^{6}-30 \rho^{4}+12 \rho^{2}-1$ & Esférica secundaria \\
\hline 16 & $q_{16}$ & $\rho^{4} \cos 4 \theta$ & Tetrafoil x \\
\hline 17 & $q_{17}$ & $\rho^{4} \sin 4 \theta$ & Tetrafoil y \\
\hline 18 & $q_{18}$ & $\left(5 \rho^{2}-4\right) \rho^{3} \cos 3 \theta$ & Trébol secundario $\mathrm{x}$ \\
\hline 19 & $q_{19}$ & $\left(5 \rho^{2}-4\right) \rho^{3} \sin 3 \theta$ & Trébol secundario y \\
\hline 20 & $q_{20}$ & $\left(15 \rho^{4}-20 \rho^{2}+6\right) \rho^{2} \cos 2 \theta$ & Astigmatismo terciario $\mathrm{x}$ \\
\hline 21 & $q_{21}$ & $\left(15 \rho^{4}-20 \rho^{2}+6\right) \rho^{2} \sin 2 \theta$ & Astigmatismo terciario y \\
\hline 22 & $q_{22}$ & $\left(35 \rho^{6}-60 \rho^{4}+30 \rho^{2}-4\right) \rho \cos \theta$ & Coma terciaria $\mathrm{x}$ \\
\hline 23 & $q_{23}$ & $\left(35 \rho^{6}-60 \rho^{4}+30 \rho^{2}-4\right) \rho \sin \theta$ & Coma terciaria y \\
\hline 24 & $q_{24}$ & $70 \rho^{8}-140 \rho^{6}+90 \rho^{4}-20 \rho^{2}+1$ & Esférica terciaria \\
\hline 25 & $q_{25}$ & $\rho^{5} \cos 5 \theta$ & Pentafoil x \\
\hline 26 & $q_{26}$ & $\rho^{5} \sin 5 \theta$ & Pentafoil y \\
\hline 27 & $q_{27}$ & $\left(6 \rho^{2}-5\right) \rho^{4} \cos 4 \theta$ & Tetrafoil secundario $\mathrm{x}$ \\
\hline 28 & $q_{28}$ & $\left(6 \rho^{2}-5\right) \rho^{4} \sin 4 \theta$ & Tetrafoil secundario y \\
\hline 29 & $q_{29}$ & $\left(21 \rho^{4}-30 \rho^{2}+10\right) \rho^{3} \cos 3 \theta$ & Trébol terciario $\mathrm{x}$ \\
\hline 30 & $q_{30}$ & $\left(21 \rho^{4}-30 \rho^{2}+10\right) \rho^{3} \sin 3 \theta$ & Trébol terciario y \\
\hline 31 & $q_{31}$ & $\left(56 \rho^{6}-105 \rho^{4}+60 \rho^{2}-10\right) \rho^{2} \cos 2 \theta$ & Astigmatismo cuaternario $\mathrm{x}$ \\
\hline 32 & $q_{32}$ & $\left(56 \rho^{6}-105 \rho^{4}+60 \rho^{2}-10\right) \rho^{2} \sin 2 \theta$ & Astigmatismo cuaternario y \\
\hline 33 & $q_{33}$ & $\left(126 \rho^{8}-280 \rho^{6}+210 \rho^{4}+60 \rho^{2}+5\right) \rho \cos \theta$ & Coma cuaternaria $x$ \\
\hline 34 & $q_{34}$ & $\left(126 \rho^{8}-280 \rho^{6}+210 \rho^{4}+60 \rho^{2}+5\right) \rho \sin \theta$ & Coma cuaternaria y \\
\hline 35 & $q_{35}$ & $252 \rho^{10}-630 \rho^{8}+560 \rho^{6}-210 \rho^{4}+30 \rho^{2}-1$ & Esférica cuaternaria \\
\hline
\end{tabular}

haciendo girar la rueda de la ruleta en $\mu / 2$ ocasiones. En cada ocasión existe una alta probabilidad de que un individuo $b_{k}(0)$ sea seleccionado si su valor de aptitud $\varphi_{1_{k}}$ es bueno, tal y como puede observarse en la Fig. (2).
Paso 5: Una versión mejorada del proceso de recombinación de un solo punto de cruza es aplicada a cada pareja de padres. El proceso inicia seleccionando aleatoriamente un punto de cruza $\alpha$ para la primera pareja. El punto de cruza es obteni- 
do considerando las 6 posiciones que definen la longitud del vector $a_{k}$ expresado en la Ec. (7), es decir, $\alpha$ es generada seleccionando aleatoriamente una de las 6 posiciones. De esta forma el primer par de padres, con su punto de cruza, es definido como sigue:

$$
\begin{aligned}
b_{k}(0)= & {\left[x_{k, 1}, x_{k, 2}, x_{k, \alpha}, x_{k, \alpha+1}, x_{k, \alpha+2}, x_{k, \alpha+3}\right], } \\
b_{k+1}(0)= & {\left[x_{k+1,1}, x_{k+1,2}, x_{k+1, \alpha},\right.} \\
& \left.x_{k+1, \alpha+1}, x_{k+1, \alpha+2}, x_{k+1, \alpha+3},\right]
\end{aligned}
$$

El par de coeficientes, seleccionados por $\alpha$, son combinados para formar los nuevos coeficientes que aparecerán en la pareja de hijos:

$$
\begin{aligned}
& x_{\alpha_{a}}=x_{k, \alpha}-\beta\left[x_{k, \alpha}-x_{k+1, \alpha}\right], \\
& x_{\alpha_{b}}=x_{k+1, \alpha}+\beta\left[x_{k, \alpha}-x_{k+1, \alpha}\right] .
\end{aligned}
$$

En donde $\beta$ es un número aleatorio entre 0 y 1 . El paso final es completar el proceso de recombinación, entre $b_{k}(0) \mathrm{y}$ $b_{k+1}(0)$, intercambiando sus coeficientes de aberración que están a la derecha del punto de cruza $\alpha$ :

$$
\begin{aligned}
c_{k}(0)= & {\left[x_{k, 1}, x_{k, 2}, x_{\alpha_{a}},\right.} \\
& \left.x_{k+1, \alpha+1}, x_{k+1, \alpha+2}, x_{k+1, \alpha+3}\right], \\
c_{k+1}(0)= & {\left[x_{k+1,1}, x_{k+1,2}, x_{\alpha_{b}},\right.} \\
& \left.x_{k, \alpha+1}, x_{k, \alpha+2}, x_{k, \alpha+3}\right] .
\end{aligned}
$$

Si $\alpha=1$, entonces solo los coeficientes de la derecha, de los dos individuos seleccionados, son intercambiados. Por otro lado, si $\alpha=6$, entonces solo los coeficientes de la izquierda, en el par de padres seleccionados, son intercambiados. Este tipo de proceso de recombinación no permite que los valores en los coeficientes, del par de hijos obtenidos en las Ecs. (13) y (14), queden fuera los límites establecidos por los respectivos padres, a menos que $\beta>1$ [12]. Además, el problema de que no se introduzca nueva información es evitado mediante el uso de las Ecs. (11) y (12). En el proceso de recombinación con un solo punto de cruza, la población inicial $\mathcal{P}_{\text {padres }}(0)$ es siempre propagada a las siguientes generaciones, solo que en diferentes combinaciones [19].

Finalmente, el paso 5 es aplicado a las parejas restantes para formar la población de hijos $\mathcal{P}_{\text {hijos }}(0)$.

Paso 6: $\mathrm{Si}$ en las poblaciones de $\mathcal{P}_{\text {padres }}(0)$ y $\mathcal{P}_{\text {hijos }}(0)$ son encontrados individuos duplicados (clones), entonces el proceso de mutación es aplicado. El proceso de mutación consiste en sumar a un individuo duplicado un vector $m$ de $1 \times 6$ cuyos elementos corresponden a números aleatorios normalmente distribuidos, es decir:

$$
\operatorname{clon}_{k}=\left[x_{k, 1}+m_{k, 1}, x_{k, 2}+m_{k, 2}, \ldots, x_{k, 6}+m_{k, 6}\right] .
$$

Paso 7: Una nueva población de padres e hijos es generada en $\mathscr{g}=\mathscr{g}+1$. Dicha población es incluida en la siguiente generación retornando al paso 2 . Si el individuo $a_{k_{o p t}}$ es obtenido a partir de la nueva población, entonces la solución más cercana al máximo global es encontrada evitando una convergencia prematura a un máximo local. En caso contrario, el proceso de optimización continua a través de la generación de nuevos individuos seleccionando aquellos con el menor error hasta que $a_{k_{\text {opt }}}$ es encontrado o el número de generaciones previamente establecido es realizado.

Con la finalidad de implementar el experimento numérico comparativo, el segundo AE propuesto para minimizar la Ec. (5) está basado en una estrategia evolutiva (EE) cuyos procesos estocásticos se combinan con un proceso de regresión lineal ponderada localmente (RLPL). En la minimización de la Ec. (5), los datos de entrada a la EE son también definidos por $I_{\mathrm{ec}}$, mientras que el individuo encontrado $a_{k_{\mathrm{opt}}}$ corresponde a los valores de los coeficientes de los primeros 35 PZ mostrados en la Tabla I, en donde los términos de Zernike del 1 al 35 representan a las aberraciones primarias, secundarias, terciarias y cuaternarias acorde con la descripción proporcionada por Wyant et al. [10]. De esta forma, el individuo $a_{k_{\mathrm{opt}}}$ define a la función $D C O_{\mathrm{opt}}$ que mejor se relaciona a la información codificada en $I_{\mathrm{ec}}$.

Una descripción detallada de la minimización de la Ec. (5) mediante el uso de la EE es proporcionada en [13]. Además, en [20] es mostrada la eficacia de la misma configuración EE en la solución de un problema inverso relacionado al esparcimiento de la luz.

Una descripción de la EE es proporcionada a continuación, con la finalidad de especificar las características particulares que poseen sus procesos estocásticos en la minimización de la Ec. (5):

Paso 1: Una población inicial de individuos, $\mathscr{g}=0$, es generada aleatoriamente:

$$
\mathcal{P}_{\text {progenitores }}(0)=\left\{a_{1}(0), \ldots \ldots, a_{\mu}(0)\right\}
$$

en donde cada individuo $a_{k}$ es formado por un vector que contiene elementos llamados variables objeto y en donde cada variable está relacionada a un parámetro estratégico, el cual es requerido para llevar a cabo el proceso de mutación. Una variable objeto es representada por $x_{k, i}$, mientras que su correspondiente parámetro estratégico es expresado como $\sigma_{k, i}$. Por lo tanto, los 35 coeficientes de aberración de la Tabla I son representados en las variables objeto de un individuo $a_{k}$ a través de la siguiente expresión:

$$
\begin{aligned}
a_{k} & =\left[x_{k, 1}, \ldots \ldots, x_{k, 35}, \sigma_{k, 1}, \ldots \ldots, \sigma_{k, 35}\right], \\
k & \in\{1, \ldots \ldots, \mu\}
\end{aligned}
$$

Paso 2: La aptitud $\varphi_{2_{k}}$ de cada progenitor $a_{k}$ es evaluada con la función objetivo $D E\left(I_{k}, I_{e c}\right)$ expresada en la Ec. (5).

Paso 3: Los primeros 15 individuos de la población inicial son seleccionados en una subpoblación, nombrada $\mathcal{P}_{\text {cruza }}(0)$, la cual es requerida para llevar a cabo los procesos de recombinación, y así generar el 30 \% de la población de descendientes, mientras que el resto de la subpoblación de 35 individuos, $\mathcal{P}_{\text {mutación }}(0)$, es considerada en el proceso de mutación para formar el $70 \%$ restante de la población de descendientes. 


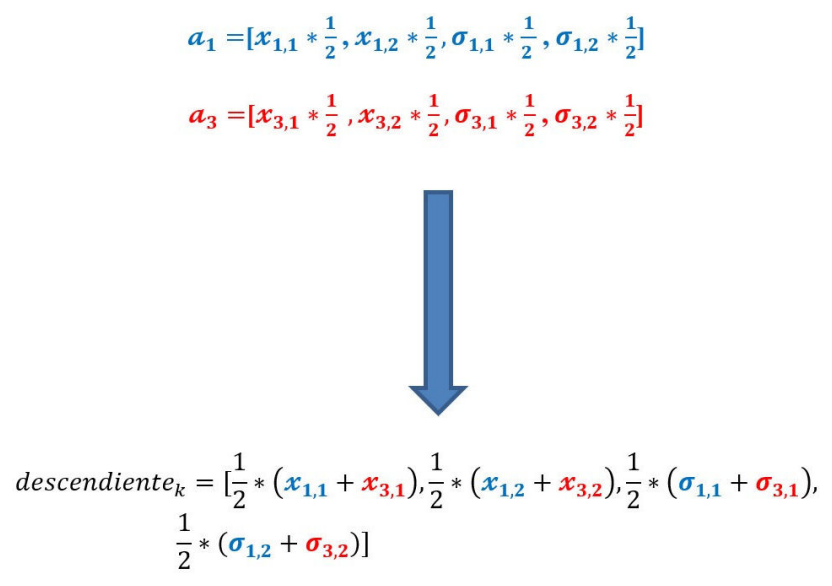

FIGURA 3. Ejemplo que ilustra el uso de la recombinación intermedia local (RIL) en un par de progenitores con un conjunto indexado de 4 elementos cada uno. La RIL trabaja seleccionando aleatoriamente un par de progenitores y calcula una suma ponderada de los correspondientes elementos de los dos progenitores.

Paso 4: La recombinación intermedia local (RIL) es usada para producir el $10 \%$ de población de descendientes. La RIL opera seleccionando aleatoriamente a un par de progenitores del subconjunto $\mathcal{P}_{\text {cruza }}(0)$, con la finalidad de que el valor de cualquier variable objeto y parámetro estratégico de un descendiente reciba una contribución de ambos progenitores seleccionados. La RIL es definida por:

$$
c_{\mathrm{cruza}_{k}}=r_{k} a_{\gamma_{1}}+\left(1-r_{k}\right) a_{\gamma_{2}}
$$

en donde $r_{k}$ es un número aleatorio en el intervalo $[0,1]$ o $r_{k}=1 / 2$ y $\left(\gamma_{1}, \gamma_{2}\right) \sim \cup(1, \ldots,[\mu / 3.33333333])$ para cada descendiente. En la Fig. 3 se muestra un ejemplo ilustrativo de la RIL.

Paso 5: La recombinación discreta (RD) es usada para producir un $20 \%$ de población de descendientes. La RD trabaja seleccionando aleatoriamente a un par de progenitores del subconjunto $\mathcal{P}_{\text {cruza }}(0)$. Debido a que cada progenitor es definido como el vector de un conjunto de 70 elementos indexados, por consiguiente, el proceso RD contempla 4900 posibles descendientes que resultarían de la combinación de los elementos de los dos progenitores. De esta forma, la RD selecciona al azar a dos descendientes, donde cada uno de ellos podría inclusive ser cualquiera de los dos padres. Por lo tanto, la $\mathrm{RD}$ puede ser expresada de la siguiente manera:

$$
c_{\mathrm{cruza} 2_{k}}=a_{\eta_{1}} \sigma a_{\eta_{2}}
$$

donde $\left(\eta_{1}, \eta_{2}\right) \sim \cup(\{1, \ldots,[\mu / 3.33333333]\})$ al azar para cada $\eta$. Un ejemplo ilustrativo de la RD es mostrado en la Fig. 4.

Paso 6: El proceso de mutación es aplicado a la subpoblación $\mathcal{P}_{\text {mutación }}(0)$ para producir el $70 \%$ de la población de descendientes. El proceso de mutación genera cambios aleatorios en cada progenitor sumando a cada variable objecto $x_{k, i}$ su correspondiente variable aleatoria, la cual es tomada de una distribución normal con media igual a cero y desviación estándar igual a $\sigma_{k, i}$, es decir:

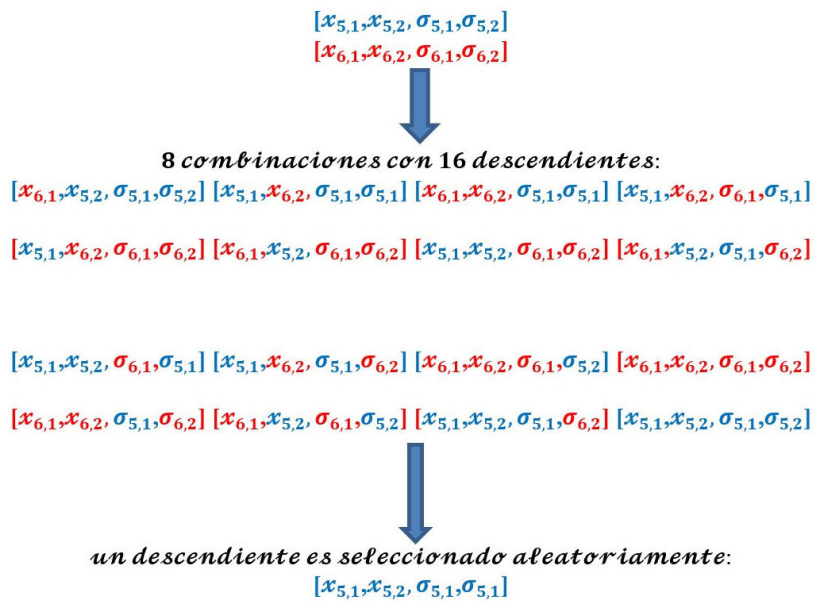

FIGURA 4. Ejemplo que ilustra el uso de la recombinación discreta $(\mathrm{RD})$ en un par de progenitores con un conjunto indexado de 4 elementos cada uno. De las 16 combinaciones posibles, la RD selecciona un descendiente al azar.

$$
\begin{aligned}
c_{\text {muta }_{k}} & =\left[x_{k, 1}+\mathcal{M}\left(0, \sigma_{k, 1}\right),+x_{k, 2}+\mathcal{M}\left(0, \sigma_{k, 2}\right),\right. \\
& \left.\ldots \ldots, x_{k, 35}+\mathcal{M}\left(0, \sigma_{k, 35}\right)\right] .
\end{aligned}
$$

Paso 6: Una vez generada la población $\mathcal{P}_{\text {descendientes }}(0)$, la aptitud $\varphi_{2_{\mu+k}}$ de cada descendiente $c_{k}$ es evaluada con la función objetivo $D E\left(I_{k}, I_{e c}\right)$ expresada en la Ec. (5).

Paso 7: El proceso de selección escoge a los mejores $\mu$ individuos de la población resultante de progenitores y descendientes, $2 \mu$, prescindiendo del resto de la población. Las aptitudes de los mejores $\mu$ individuos son agrupadas en orden ascendente en un vector fila, del menor valor $\varphi_{2_{1}}$ al mayor valor $\varphi_{2_{\mu}}$ :

$$
\text { aptitudes }=\left[\varphi_{2_{1}}, \ldots \ldots, \varphi_{2_{\mu}}\right]
$$

Paso 8: Partiendo de la población de los mejores $\mu$ descendientes, los individuos que resulten del proceso de mutación son considerados para una actualización de sus correspondientes parámetros estratégicos. Dicha actualización, conocida como la regla del éxito 1/5, establece que si la probabilidad estimada de una mutación exitosa es $>1 / 5$, entonces los parámetros estratégicos, del correspondiente individuo mutado, son incrementados; de lo contrario son disminuidos, es decir:

$$
\begin{aligned}
& S i\left[\varphi_{2}\left(a_{\text {mutado }_{k}}\right)>\varphi_{2}\left(a_{\text {seleccionado }_{k}}\right)\right]>\frac{1}{5}, \\
& \sigma_{\text {actualizado }_{k, i}}=\sigma_{\text {mutado }_{k, i}} *(1+l), \quad i=1, \ldots \ldots, 35
\end{aligned}
$$

de lo contrario

$$
\sigma_{\text {actualizado }_{k, i}}=\frac{\sigma_{\text {mutado }_{k, i}}}{l}
$$

donde $l$ es una constante positiva. En los experimentos numéricos que serán realizados en el presente trabajo $l=$ 
0.001. Además, $a_{\text {seleccionado }_{k}}$ es uno de los individuos en $\mathcal{P}_{\text {mutación }}(0)$, siendo este el antecesor de $a_{\text {mutado }_{k}}$.

Es importante agregar que la actualización dinámica de los parámetros estratégicos de los descendientes encontrados a través del proceso de mutación es en respuesta a las características del espacio de muestreo explorado.

Paso 9: La población resultante en el paso 8 es usada por el algoritmo RLPL para crear un modelo lineal local con la finalidad de predecir otro conjunto de individuos, $\mathcal{P}_{\text {progenitores }}(\mathscr{g}+$ $1)=\left\{a_{1}(g+1), \ldots \ldots, a_{\mu}(g+1)\right\}$, solo en una región alrededor del punto de consulta $I_{\text {ec }}$ de la Ec. (5). Así, el algoritmo RLPL selecciona un mejor punto en la vecindad de cada individuo de la población obtenida por la $\mathrm{EE}$ y produce la siguiente generación, $g=g+1$, retornando al paso 2 .

Si el individuo $a_{k_{\mathrm{opt}}}$ es encontrado por la EE partiendo de la población generada por el algoritmo RLPL, entonces la solución más cercana al mínimo global es encontrada evitando una convergencia prematura a un mínimo local. En caso contrario, el proceso de optimización continua a través de la generación de nuevos individuos seleccionando aquellos con el menor error hasta que la solución más cercana al mínimo global es encontrada o el número de generaciones previamente establecido es realizado.

\section{Resultados numéricos}

Nuestros estudios numéricos comparativos consisten en el análisis de dos interferogramas reales cuyas DCOs son generadas en una apertura circular mediante el uso de un interferómetro Fizeau.

Con la finalidad de verificar la robustez y precisión del AG y de la EE, nosotros consideramos inicialmente el análisis de un interferograma con franjas cerradas en la prueba de una superficie óptica esférica y posteriormente el análisis de un interferograma con franjas rectas en la prueba de un plano óptico. Ambos algoritmos son implementados en Fortran y los códigos son ejecutados en un ordenador portátil con un procesador Intel Core ${ }^{\mathrm{TM}}$ i7 de $2.3 \mathrm{GHz}$ y memoria RAM de 12 GB. Debido a que cada $D C O$ a ser reconstruida se da en una apertura circular, nosotros hemos considerado la formulación de Kingslake y a los $\mathrm{PZ}$ como funciones $D C O$ para ser usados por el AG y la EE, respectivamente. Además, cada interferograma a ser analizado es obtenido minimizando la presencia del tilt y el defoco mencionados en la Ec. (2) y la Tabla I, ya que sus respectivos coeficientes no expresan ningún tipo de aberración relacionada a la $D C O$, únicamente determinan los errores de posicionamiento, de inclinación y traslación, que pueden tener las componentes del arreglo óptico interferométrico, tales como las superficies ópticas de prueba y de referencia.
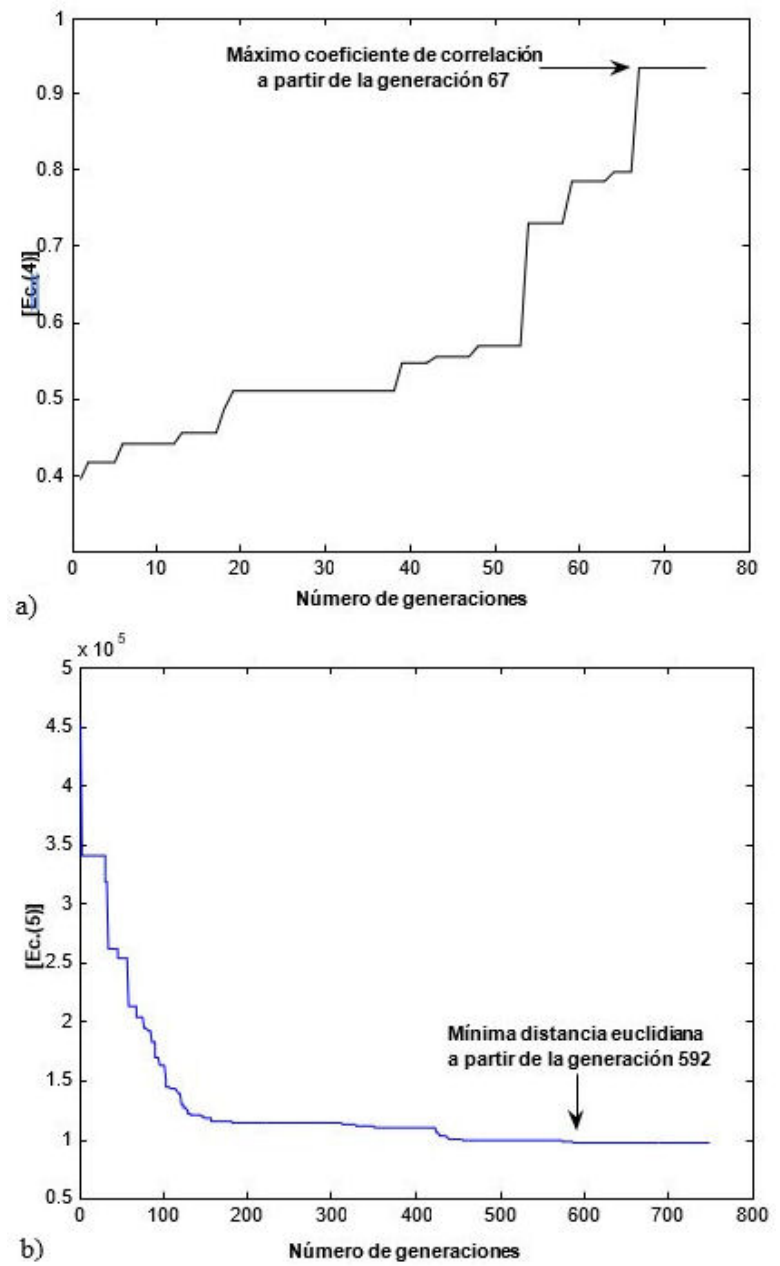

FIGURA 5 Procesos de optimización realizados por los algoritmos evolutivos propuestos. a) Maximización de la Ec. (4) mediante el uso de un AG. b) Minimización de la Ec. (5) a través de la EE. El desempeño de cada algoritmo es obtenido usando una población inicial de 3000 y 50 individuos, respectivamente.

TABLA II. Valores calculados de los coeficientes de la formulación de Kingslake. a) En el análisis del primer interferograma real. b) En el análisis del segundo interferograma real. Los valores de los coeficientes son expresados como múltiplos de la longitud de onda usada $(\lambda=632.8 \mathrm{~nm})$.

\begin{tabular}{cccc}
\hline Término & a.- Valor del coeficiente de aberración & b.- Valor del coeficiente de aberración & Descripción del término \\
\hline 1 & $-2.1982 \lambda$ & $2.9703 \lambda$ & Tilt x \\
2 & $-0.4583 \lambda$ & $0.0712 \lambda$ & Tilt y \\
3 & $1.3282 \lambda$ & $-0.1641 \lambda$ & Defoco \\
4 & $0.0641 \lambda$ & $0.0179 \lambda$ & Astigmatismo \\
5 & $-0.0009 \lambda$ & $-0.0983 \lambda$ & Coma \\
6 & $-0.2299 \lambda$ & $0.0865 \lambda$ & Esférica \\
\hline
\end{tabular}



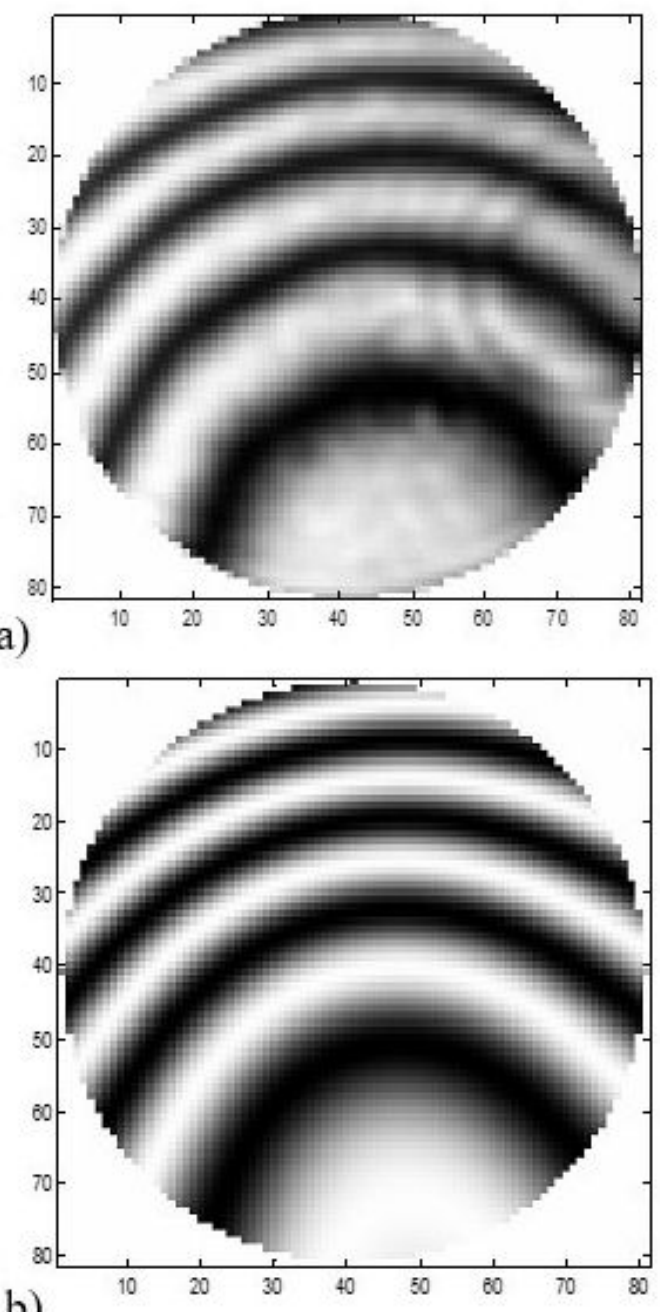

b)

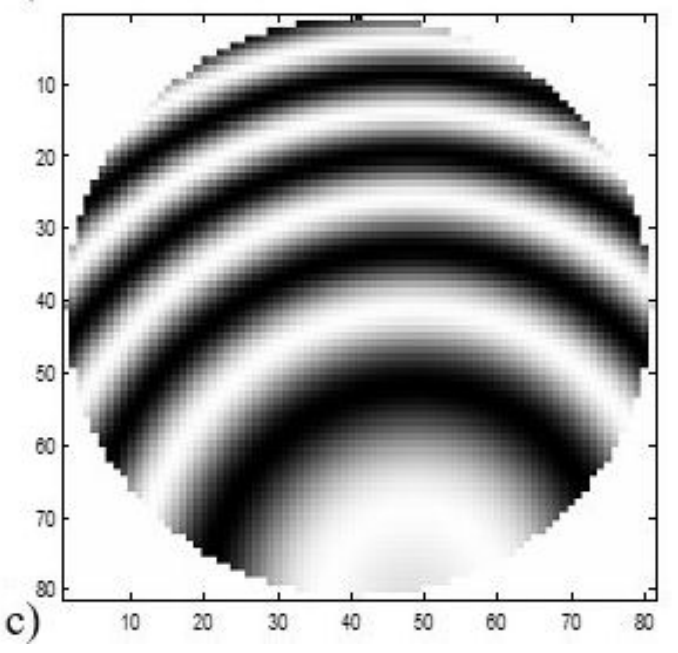

FIGURA 6. Interferograma real analizado durante el primer estudio numérico comparativo. a) Interferograma real con franjas cerradas. b) Interferograma $I_{1_{\text {opt }}}$ optimizado con los valores de los coeficientes de la formulación de Kingslake correspondientes a la solución $\operatorname{CORR}\left(I_{1_{\mathrm{opt}}}, I_{e c}\right)$ para el máximo coeficiente de correlación $\varphi_{1_{1_{\text {opt }}}}$ en la Ec. (4). c) Interferograma $I_{1_{\text {opt }}}$ optimizado con los valores de los coeficientes de los PZ relacionados a la solución $D E\left(I_{1_{\mathrm{opt}}}, I_{e c}\right)$ para la mínima distancia euclidiana $\varphi_{2_{1_{\text {opt }}}}$ en la Ec. (5). a)

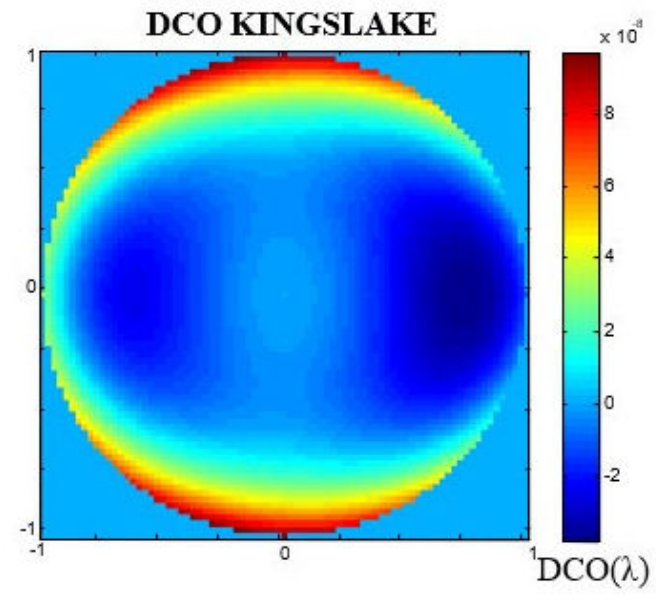

b)
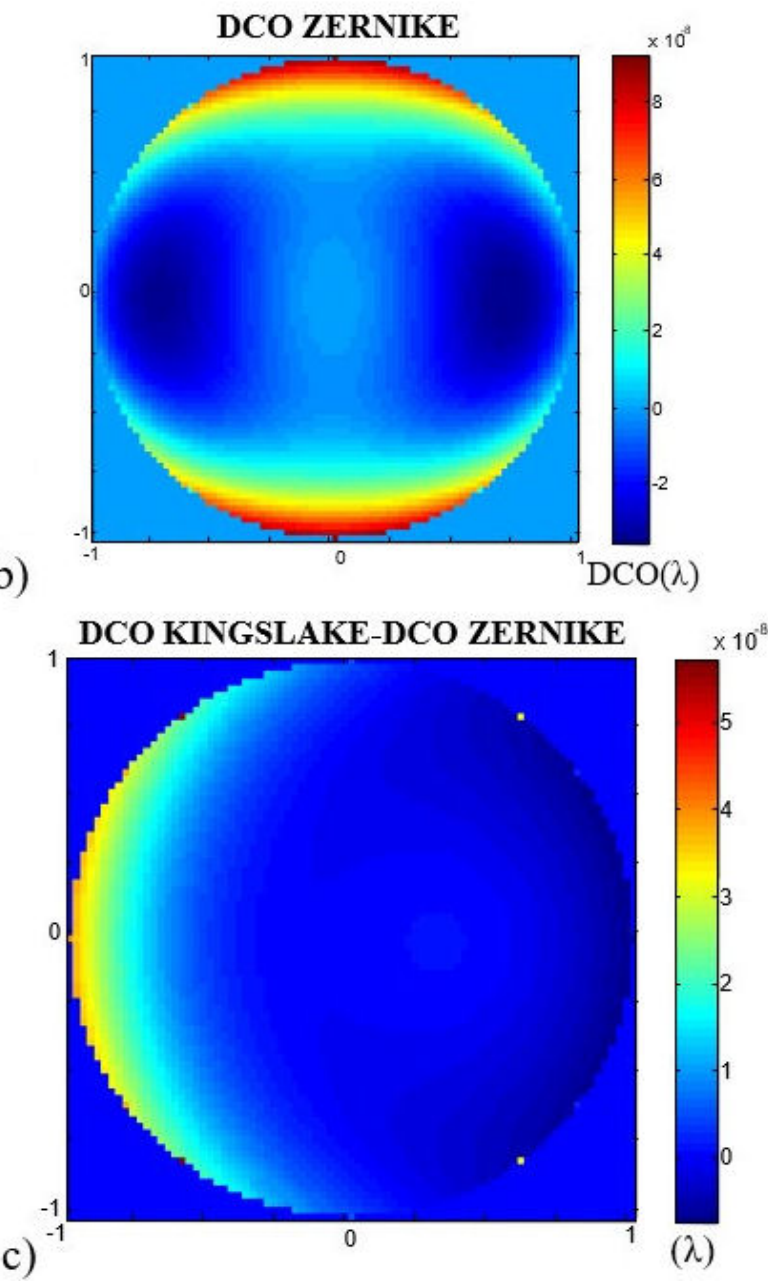

FIgURA 7. Mapas $D C O_{\text {opt }}$ reconstruidos en el primer estudio numérico comparativo. a) Reconstrucción con el AG. b) Reconstrucción con la EE. c) Diferencia entre los dos mapas. Cada mapa $D C O_{\text {opt }}$ es generado removiendo sus respectivos errores de posicionamiento. En b), el término de pistón también es removido.

En la Fig. 5 son mostrados los resultados del primer estudio numérico comparativo que demuestran que la $D C O$ del interferograma real en la Fig. 6a) puede ser reconstruida, ya sea maximizando la Ec. (4) con el AG o minimizando la Ec. (5) 
TABLA III. Valores calculados de los coeficientes de los polinomios de Zernike. a) En el análisis del primer interferograma real. b) En el análisis del segundo interferograma real. Los valores de los coeficientes son expresados como múltiplos de la longitud de onda usada $(\lambda=632.8 \mathrm{~nm})$.

\begin{tabular}{|c|c|c|c|}
\hline Término & a.- Valor del coeficiente de aberración & b.- Valor del coeficiente de aberración & Descripción del término \\
\hline 0 & $0.8468 \lambda$ & $0.4617 \lambda$ & Pistón \\
\hline 1 & $2.2424 \lambda$ & $2.9705 \lambda$ & Tilt $\mathrm{x}$ \\
\hline 2 & $0.4576 \lambda$ & $0.0054 \lambda$ & Tilt y \\
\hline 3 & $-0.5884 \lambda$ & $-0.0084 \lambda$ & Defoco \\
\hline 4 & $-0.0817 \lambda$ & $0.0186 \lambda$ & Astigmatismo $\mathrm{x}$ \\
\hline 5 & $0.0070 \lambda$ & $0.0031 \lambda$ & Astigmatismo y \\
\hline 6 & $0.0097 \lambda$ & $0.0086 \lambda$ & Coma x \\
\hline 7 & $-0.0079 \lambda$ & $-0.0248 \lambda$ & Coma y \\
\hline 8 & $0.0007 \lambda$ & $0.0026 \lambda$ & Esférica primaria \\
\hline 9 & $0.0000 \lambda$ & $0.0000 \lambda$ & Trébol x \\
\hline 10 & $0.0000 \lambda$ & $0.0000 \lambda$ & Trébol y \\
\hline 11 & $0.0000 \lambda$ & $0.0000 \lambda$ & Astigmatismo secundario $\mathrm{x}$ \\
\hline 12 & $0.0000 \lambda$ & $0.0000 \lambda$ & Astigmatismo secundario y \\
\hline 13 & $0.0001 \lambda$ & $0.0000 \lambda$ & Coma secundaria $\mathrm{x}$ \\
\hline 14 & $0.0000 \lambda$ & $0.0000 \lambda$ & Coma secundaria y \\
\hline 15 & $0.0000 \lambda$ & $0.0000 \lambda$ & Esférica secundaria \\
\hline 16 & $0.0000 \lambda$ & $0.0000 \lambda$ & Tetrafoil x \\
\hline 17 & $0.0000 \lambda$ & $0.0000 \lambda$ & Tetrafoil y \\
\hline 18 & $0.0000 \lambda$ & $0.0000 \lambda$ & Trébol secundario $\mathrm{x}$ \\
\hline 19 & 0.0000 & $0.0000 \lambda$ & Trébol secundario y \\
\hline 20 & $0.0000 \lambda$ & $0.0000 \lambda$ & Astigmatismo terciario $\mathrm{x}$ \\
\hline 21 & $0.0000 \lambda$ & $0.0000 \lambda$ & Astigmatismo terciario y \\
\hline 22 & $0.0000 \lambda$ & $0.0000 \lambda$ & Coma terciaria $\mathrm{x}$ \\
\hline 23 & $0.0000 \lambda$ & $0.0000 \lambda$ & Coma terciaria y \\
\hline 24 & $0.0000 \lambda$ & $-0.0000 \lambda$ & Esférica terciaria \\
\hline 25 & $0.0000 \lambda$ & $0.0000 \lambda$ & Pentafoil x \\
\hline 26 & $0.0000 \lambda$ & $0.0000 \lambda$ & Pentafoil y \\
\hline 27 & $0.0000 \lambda$ & $0.0000 \lambda$ & Tetrafoil secundario $\mathrm{x}$ \\
\hline 28 & $0.0000 \lambda$ & $0.0000 \lambda$ & Tetrafoil secundario y \\
\hline 29 & $0.0000 \lambda$ & $0.0000 \lambda$ & Trébol terciario $\mathrm{x}$ \\
\hline 30 & $0.0000 \lambda$ & $0.0000 \lambda$ & Trébol terciario y \\
\hline 31 & $0.0000 \lambda$ & $0.0000 \lambda$ & Astigmatismo cuaternario $\mathrm{x}$ \\
\hline 32 & $0.0000 \lambda$ & $0.0000 \lambda$ & Astigmatismo cuaternario y \\
\hline 33 & $0.0000 \lambda$ & $0.0000 \lambda$ & Coma cuaternaria $\mathrm{x}$ \\
\hline 34 & $0.0000 \lambda$ & $0.0000 \lambda$ & Coma cuaternaria y \\
\hline 35 & $0.0000 \lambda$ & $0.0000 \lambda$ & Esférica cuaternaria \\
\hline
\end{tabular}

a través de la EE. En la Fig. 5a) es mostrado el proceso de maximización de la Ec. (4) cuya gráfica corresponde con el valor de aptitud $\varphi_{1_{1}}$ del mejor individuo encontrado, desde la generación 1 hasta la generación 75, mientras que la Fig. 5b) muestra el proceso de minimización de la Ec. (5) donde la gráfica corresponde con el valor de aptitud $\varphi_{2_{1}}$ del mejor in- dividuo obtenido, desde la generación 1 hasta la generación 750 .

En la Fig. 5a), el máximo coeficiente de correlación $\left(\boldsymbol{\varphi}_{\mathbf{1}_{\mathbf{1}} \text { opt }}\right)$ es obtenido cuando el AG encuentra la solución más cercana al máximo global $\left(a_{\mathbf{1}_{\text {opt }}}\right)$ en la generación 67 , en tanto que en la Fig. 5(b) la mínima distancia euclidiana $\left(\boldsymbol{\varphi}_{\mathbf{2}_{\mathbf{1}}}\right)$ 


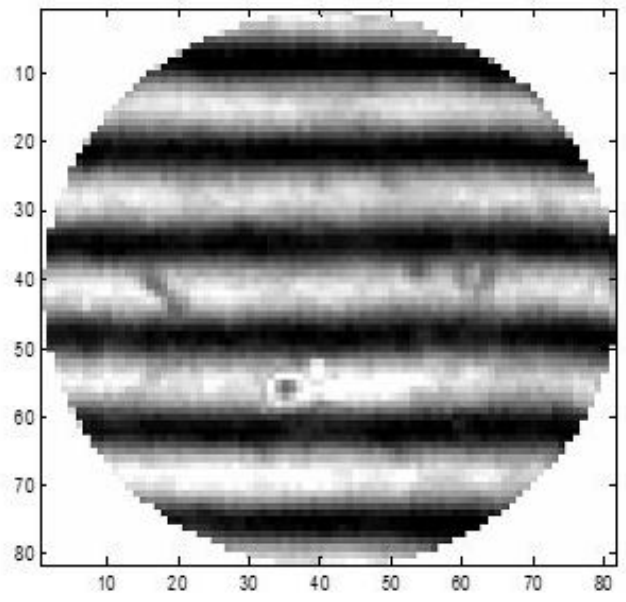

a)

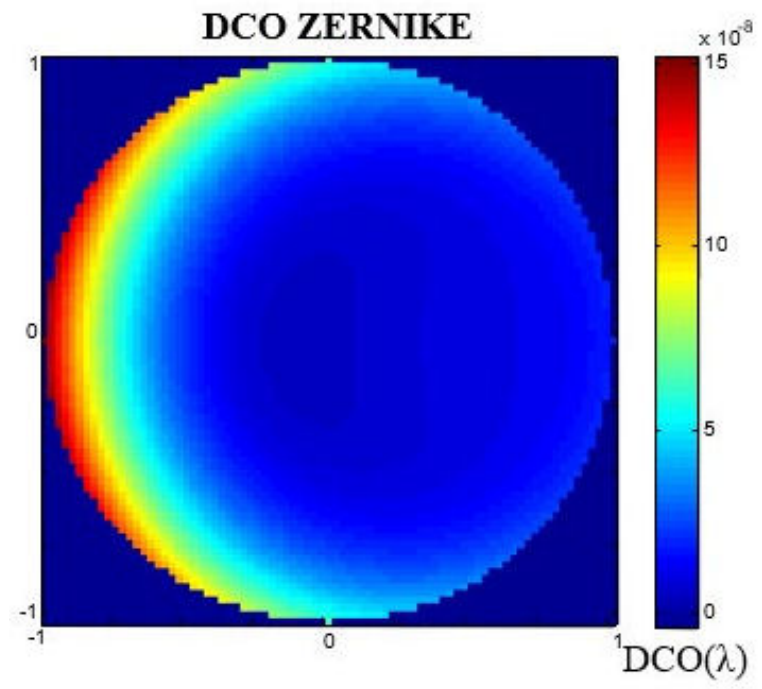

c)

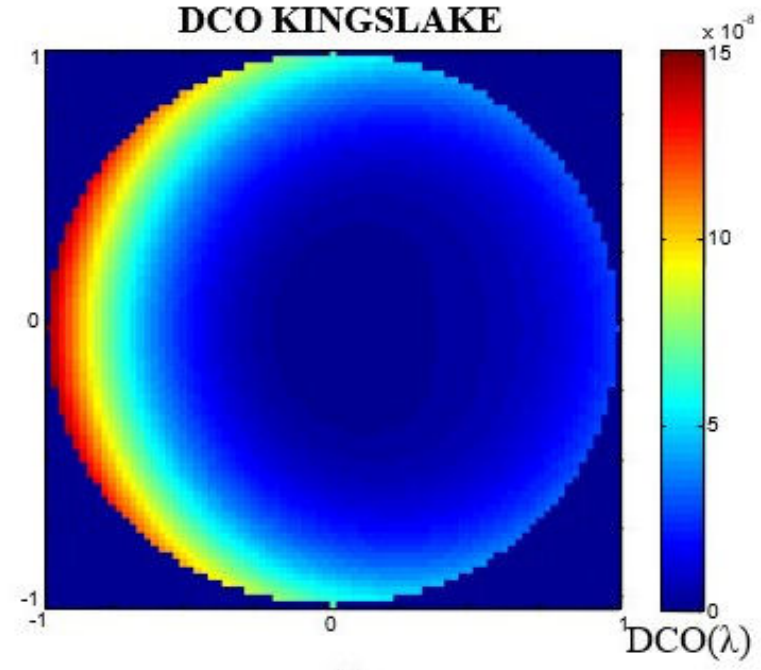

b)
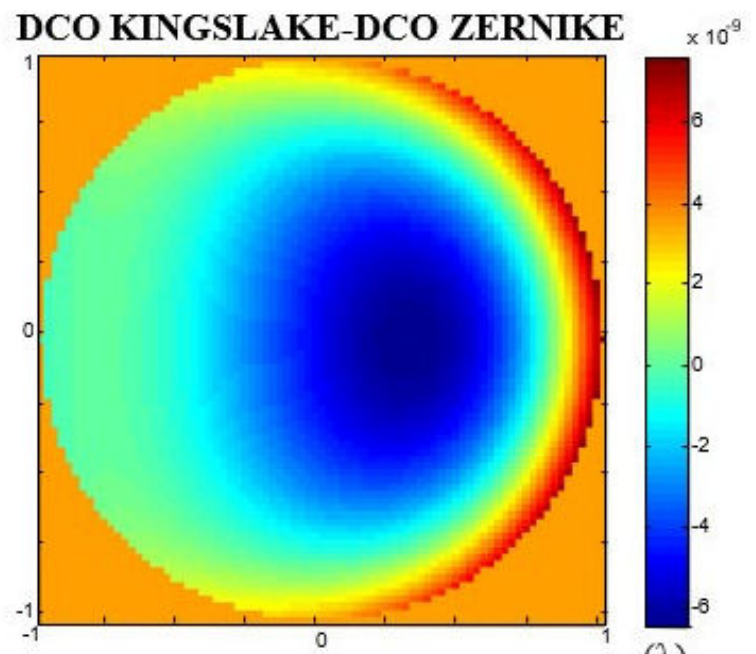

d)

FIGURA 8. Interferograma real analizado y sus mapas $D C O_{\text {opt }}$ reconstruidos en el segundo estudio numérico comparativo. a) Interferograma real con franjas rectas. b) Reconstrucción con el AG. c) Reconstrucción con la EE. d) Diferencia entre los dos mapas. Cada mapa $D C O_{\text {opt }}$ es generado removiendo sus correspondientes errores de posicionamiento. En c), el término de pistón también es removido.

es determinada cuando la EE obtiene la solución más cercana al mínimo global $\left(a_{\mathbf{1}_{\mathbf{o p t}}}\right)$ en la generación 592.

En la Tabla II(a) son mostrados los valores de los coeficientes de aberración de la formulación de Kingslake correspondientes a la solución $\operatorname{CORR}\left(I_{1_{\text {opt }}}, I_{\text {ec }}\right)$ para el máximo coeficiente de correlación $\left(\boldsymbol{\varphi}_{\mathbf{1}_{\mathbf{1}} \text { opt }}\right)$ en la Ec. (4), mientras que en la Tabla III(a) son mostrados los valores de los coeficientes de aberración de los PZ relacionados a la solución $D E\left(I_{\mathbf{1}_{\text {opt }}}, I_{\text {ec }}\right)$ para la mínima distancia euclidiana $\left(\boldsymbol{\varphi}_{\mathbf{2}_{\mathbf{1}_{\text {opt }}}}\right)$ en la Ec. (5).

Como puede ser visto en las Figs. 6b) y 6c), cada interferograma simulado $\boldsymbol{I}_{\mathbf{1}_{\text {opt }}}$ es prácticamente idéntico al primer interferograma real analizado. Consecuentemente, hay una gran similitud entre el mapa $D C O_{\text {opt }}$ dado por la solución
$\operatorname{CORR}\left(I_{1_{\mathrm{opt}}}, I_{\mathrm{ec}}\right)$ y el mapa $D C O_{\text {opt }}$ definido por la solución $D E\left(I_{1_{\text {opt }}}, I_{\text {ec }}\right)$ [ver Fig. 7(a) y 7(b), respectivamente].

En la Fig. 7c) es mostrada la diferencia entre los mapas reconstruidos. Por lo tanto, los resultados numéricos en las Figs. 6 y 7 ilustran la validez de que la DCO del interferograma real en la Fig. 6a) puede ser reconstruida con los coeficientes de aberración mostrados en la Tabla II(a) o considerando los coeficientes de aberración de la Tabla III(a).

De acuerdo con los 2 procesos de optimización mostrados en la Fig. 5, el AG tan solo necesitó 67 generaciones para encontrar el valor de aptitud $\left(\boldsymbol{\varphi}_{\mathbf{1}_{\mathbf{1}}}\right)$ en comparación con las 592 generaciones realizadas por la EE para obtener el valor de aptitud $\left(\boldsymbol{\varphi}_{\mathbf{2}_{\mathbf{1}}}\right)$. No obstante, al AG le tomó 71 segundos en encontrar la solución más cercana al máximo global, en 


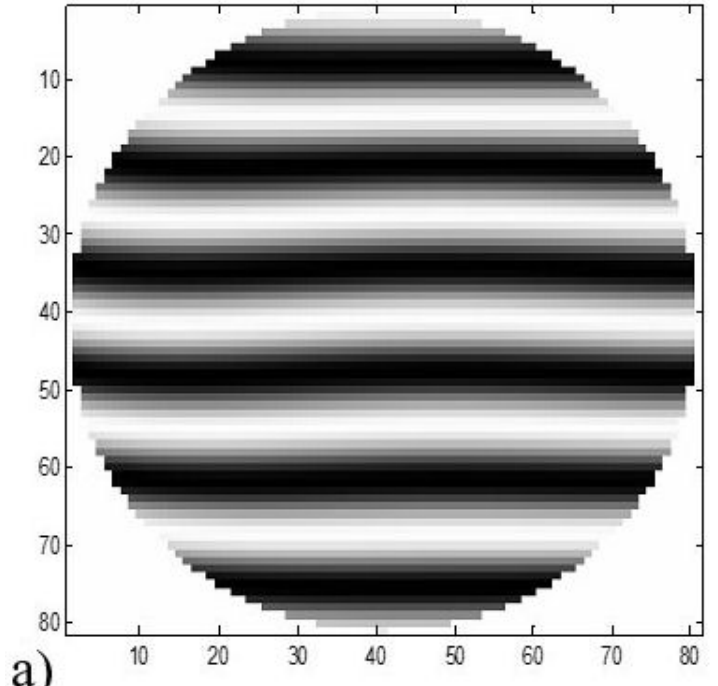

a)

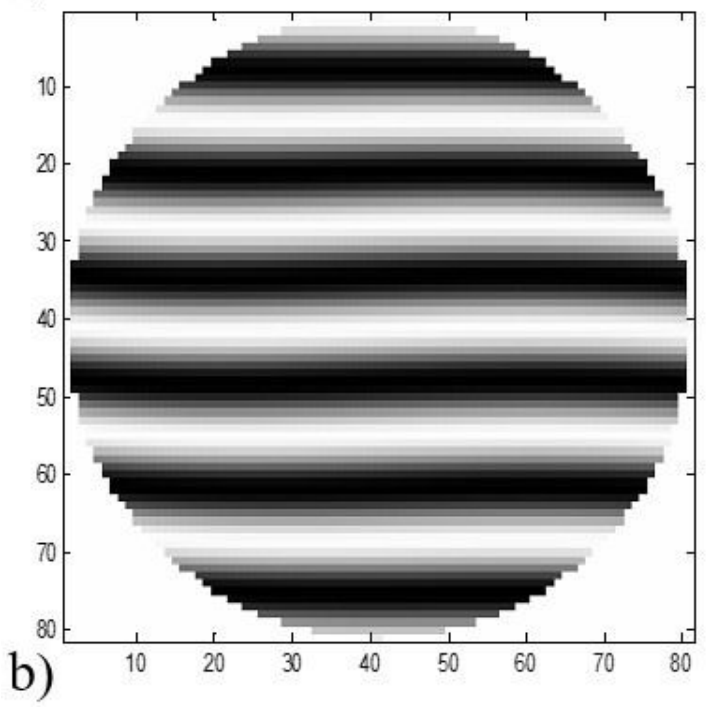

FIGURA 9. Interferogramas recuperados durante el segundo estudio numérico comparativo. a) Interferograma $I_{1_{\mathrm{opt}}}$ optimizado por la solución $\operatorname{CORR}\left(I_{1_{\mathrm{opt}}}, I_{e c}\right)$ para el máximo coeficiente de correlación $\varphi_{1_{1_{\text {opt }}}}$ en la Ec. (4). b) Interferograma $I_{1_{\mathrm{opt}}}$ optimizado por la solución $D E\left(I_{1_{\text {opt }}}, I_{e c}\right)$ para la mínima distancia euclidiana $\varphi_{2_{1_{\mathrm{opt}}}}$ en la Ec. (5).

cambio, la EE convergió a la solución más cercana al mínimo global en aproximadamente 1/9 del tiempo de ejecución del AG.

La diferencia en los tiempos de ejecución es establecida por los diferentes requerimientos de los dos AEs propuestos, es decir, para describir un comportamiento como el mostrado en la Fig. 5a), el AG requirió en cada generación de una población de individuos 60 veces mayor en comparación a la población de individuos requerida por la EE durante cada generación del proceso de optimización mostrado en la Fig. 5b).

Por lo tanto, para realizar una búsqueda eficaz en un espacio de muestreo alargado y encontrar la solución más cercana al máximo global, el AG debe ser usado con una población considerable de individuos en cada generación, 3000 individuos, con la finalidad de explotar los elementos del mayor

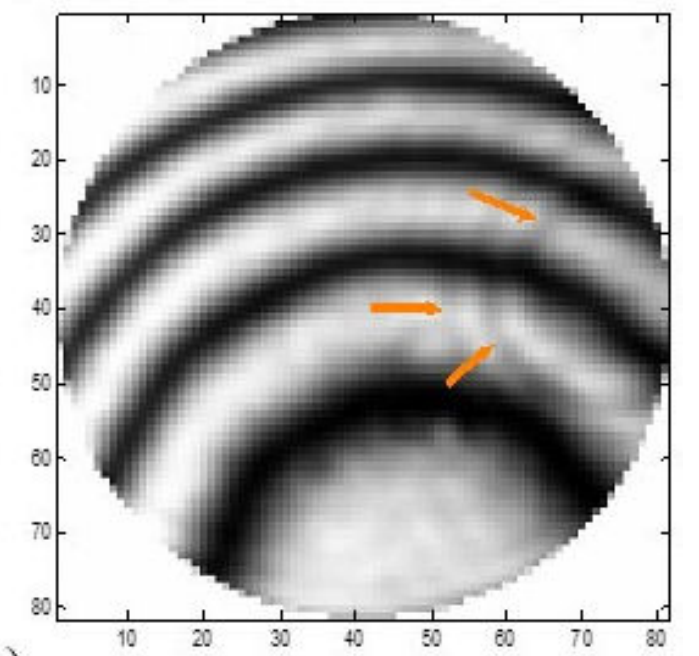

a)

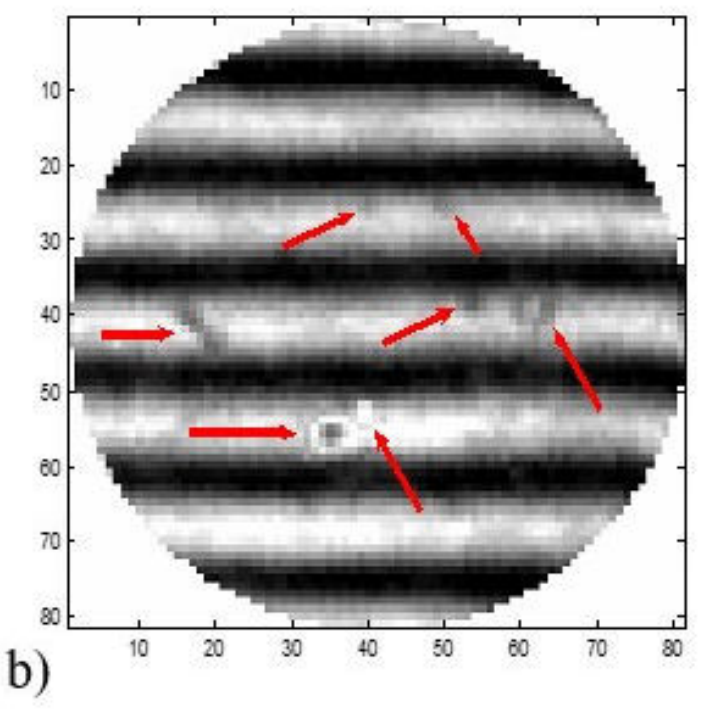

FIGURA 10. Defectos visibles en los interferogramas experimentales analizados. a) Las flechas indican que el primer interferograma real posee una degradación por la difracción de partículas de polvo en los caminos ópticos. b) Las flechas indican la degradación ocasionada por la formación del moteado en diferentes franjas del segundo interferograma real.

número posible de individuos, con buenos valores de aptitud, y propiciar la diversidad necesaria en la población a través del uso del principal proceso estocástico del algoritmo, la recombinación. Por el contrario, para realizar una búsqueda en un espacio de muestreo solo alrededor del punto consulta $I_{e c}$ de la Ec. (5), la EE tan solo requirió de 50 individuos por generación, ya que con esa cantidad de individuos el algoritmo RLPL puede ser usado para predecir a los individuos, $\mathcal{P}_{\text {progenitores }}(\mathscr{g}+1)=\left\{a_{1}(\mathscr{g}+1), \ldots \ldots, a_{\mu}(g+1)\right\}$, los cuales mantienen la diversidad necesaria para prevenir una convergencia prematura a un mínimo local y así ser utilizados por la EE en cantidad y variedad hasta encontrar la solución más cercana al mínimo global, siendo la mutación el principal proceso estocástico. 
La Fig. 8 muestra el segundo interferograma real [Fig. 8a)], cuya $D C O$ es también reconstruida de manera efectiva resolviendo las Ecs. (4) y (5) mediante el uso del AG y la EE, respectivamente. Los resultados obtenidos del segundo estudio numérico comparativo son mostrados en las Figs. 8 y 9. En las Figs. 8(b) y 8 (c) se pueden observar los mapas $D C O_{\text {opt }}$ reconstruidos a partir de los valores de los coeficientes de aberración mostrados en las Tablas II(b) y III(b). La diferencia entre los dos mapas $D C O_{\text {opt }}$, ver Fig. 8(d), hace evidente la gran similitud entre ellos. Consecuentemente, los interferogramas simulados como soluciones de $\operatorname{CORR}\left(I_{1_{\text {opt }}}, I_{\text {ec }}\right)$ y $D E\left(I_{1_{\text {opt }}}, I_{\text {ec }}\right)$ tienden a ser muy similares entre ellos, tal y como puede ser observado en las Figs. 9(a) y 9(b). Además, dichos interferogramas simulados son idénticos al interferograma real mostrado en la Fig. 8(a), ya que representan soluciones que son muy cercanas a óptimos globales. De esta forma, la robustez y precisión de ambos AEs son similares a las obtenidas en el primer estudio numérico comparativo.

En la Fig. 10 es indicada la degradación que es visible en los interferogramas reales analizados. En la Fig. 10(a) puede ser apreciada la difracción por partículas de polvo, mientras que en la Fig. 10(b) puede ser observada la degradación por ruido Speckle (moteado) cuya densidad de probabilidad obedece a una distribución uniforme.

\section{Conclusiones}

Un experimento numérico comparativo ha sido implementado para demostrar que la DCO de un interferograma real degradado por ruido puede ser reconstruida, ya sea maximizando el coeficiente de correlación mediante el uso de un AG con la formulación de Kingslake o minimizando la distancia euclidiana a través de una EE con los PZ. Los resultados numéricos mostraron no solo la robustez de ambos AEs ante la degradación de los interferogramas reales analizados, sino también la capacidad de dichos algoritmos para inhibir el impacto negativo de los términos polinomiales de más alto orden, que no son considerados en las funciones DCO durante las reconstrucciones, ya que la cantidad de términos polinomiales requeridos y los valores de los coeficientes de aberración son encontrados simultáneamente con exactitud.

\section{Agradecimientos}

Agradecemos al Mtro. José de Jesús Navarro Jr., Titular de la subdirección de investigación del Ceti, por las gestiones y facilidades otorgadas para la realización del presente trabajo.
1. J. D. Briers, Optical testing: a review and tutorial for optical engineers, Opt. Lasers Eng. 32 (1999) 111, https://doi. org/10.1016/S0143-8166(99)00062-7

2. D. Malacara, M. ServÃn y Z. Malacara, Interferogram Analysis for Optical Testing, 2da ed. (CRC Press, Boca Raton, 2005), p. 1.

3. D. Malacara-Hernández, M. Carpio-Valadéz y J. J. SánchezMondragón, Wavefront fitting with discrete orthogonal polynomials in a unit radius circle, Opt. Eng. 29 (1990) 672, https : //doi.org/10.1117/12.55629

4. J. Y. Wang y D. E. Silva, Wave-front interpretation with Zernike polynomials, Appl. Opt. 19 (1980) 1510, https://doi. org/10.1364/AO.19.001510

5. X. Yu, Y. Yao, Y. Sun y J. Tian, Stability of Zernike coefficients solved by the covariance matrix method in the analysis of the wave fron aberration, Optik 122 (2011) 1701, https: //doi.org/10.1016/j.ijleo.2010.10.028

6. X. Yu, Y. Yao, Y. Sun, J. Tian y C. Liu, The influence of the sampling dots on the analysis of the wave front aberration by using the covariance matrix method, $O p$ tik 123 (2012) 792, https://doi.org/10.1016/j. ijleo.2011.06.039

7. D. Malacara y B. J. Thompson, Handbook of Optical Engineering, 1era ed. (Marcel Dekker, Nueva York, 2011), p. 373.

8. M. Rivera, Robust phase demodulation of interferograms with open or closed fringes, J. Opt. Soc. Am. A 22 (2005) 1170, https://doi.org/10.1364/JOSAA.22.001170
9. M. B. Bernini, A. Federico y G. H. Kaufmann, Normalization of fringe patterns using the bidimensional empirical mode decomposition and the Hilbert transform, 48 (2009) 6862, https://doi.org/10.1364/AO.48.006862

10. R. R. Shannon y J. C. Wyant, Applied Optics and Optical Engineering, Vol. 11 (Academic Press, San Diego, 1992), pp. 1-53.

11. S. Vázquez-Montiel, J. J. Sánchez-Escobar y O. Fuentes, Obtaining the phase of an interferogram by use of an evolution strategy: Part I, Appl. Opt. 41 (2002) 3448, https://doi. org/10.1364/AO.41.003448.

12. J. J. Sánchez-Escobar, P. Alonso Magaña y S. VázquezMontiel, Obtaining the wavefront aberrations of a real interferogram by use of a hybrid genetic algorithm, Opt. Eng. 45 (2006) 105605, https://doi.org/10.1117/1.2360517

13. J. J. Sánchez-Escobar y L. I. Barbosa Santillán, Reconstruction of the wavefront aberration from real interferometric data using a hybrid evolutionary optimization algorithm with Zernike polynomials, Opt. Eng. 54 (2015) 094102, https: //doi.org/10.1117/1.OE.54.9.094102

14. B. Villalobos-Mendoza, D. Aguirre-Aguirre y F. GranadosAgustín, Study of the factors that affect the correlation behavior during the evaluation of interferograms, Opt. Eng. 57 (2018) 104111, https://doi.org/10.1117/1.OE. 57.10 .104111 .

15. N. S. Mera, L. Elliott y D. B. Ingham, On the use of Genetic Algorithms for Solving Ill-Posed Problems, Inverse Probl. Eng. 11 (2003) 105, https://doi.org/10.1080/ 1068276021000058473 
16. X. Liu y Y. Li, Optimal design of DFG-based wavelength conversion based on hybrid genetic algorithm, Opt. Express 11 (2003) 1677, https://doi.org/10.1364/OE.11. 001677.

17. A. Lara, G. Sánchez, C. A. Coello Coello y O. Schutze, HCS: A New Local Search Strategy for Memetic Multiobjective Evolutionary Algorithms, IEEE Trans. Evol. Comput. 14 (2010) 112, https://doi.org/10.1109/TEVC.2009.2024143

18. K. Sindhya, K. Miettinen y K. Deb, A Hybrid Framework for
Evolutionary Multi-Objective Optimization, IEEE Trans. Evol. Comput. 17 (2013) 495, https://doi.org/10.1109/ TEVC.2012.2204403

19. R. L. Haupt y S. E. Haupt, Practical Genetic Algorithms, 1era ed. (John Wiley and Sons, Nueva York, 1998), pp. 48-84.

20. J. J. Sánchez-Escobar, L. I. Barbosa-Santillán, J. Vargas-Ubera y Félix Aguilar-Valdés, Inversion method based on stochastic optimization for particle sizing, Appl. Opt. 55 (2016) 5806, https://doi.org/10.1364/AO.55.005806 